Original Research Paper

\title{
An Innovative Approach for Person Identification by Detection and Extraction of Optic Disc from Retina and Concha from Ear
}

\author{
${ }^{1}$ Malathy, C., ${ }^{2}$ A.K. Sadiq and ${ }^{1}$ K. Annapurani \\ ${ }^{I}$ Department of Computer Science and Engineering, SRM University, Kattankulathur, India \\ ${ }^{2}$ Department of Information Technology, Ministry of Higher Education, Oman
}

\author{
Article history \\ Received: $10-02-2014$ \\ Revised: 20-03-2014 \\ Accepted: 06-08-2014 \\ Corresponding Author: \\ Malathy, C. \\ Department of Computer Science \\ and Engineering, SRM University, \\ Kattankulathur, India \\ Email: malathyc03@gmail.com
}

\begin{abstract}
Person identification based on unimodal biometric system suffers from noise, intra class similarity, non universality, distinctiveness and spoof attacks. To alleviate the problem faced in unimodal biometric system biometric traits are combined in multimodal biometric system. In this study a new approach, to improve the recognition rate, reduces computational complexity and storage space is presented. Distinct method of person identification using detection and extraction of optic disc from retina and concha from ear is carried out Region Of Interest (ROI) locator which is proposed here automatically detects the optic disc either from right or left eye and extracts it. Feature level fusion of optic disc and concha is done for recognition of a person. The method is tested with ROI locator and without ROI locator on publicly available databases and the experimental result shows that our multimodal biometric system outperforms with ROI locator than Without ROI locator. Matching Rate (MR) of 95 to $100 \%$ and Equal Error Rate (EER) of less than $10 \%$ is achieved with this system. The new approach was tested for unimodal system with ROI locator and was able to achieve $100 \%$ Matching Rate.
\end{abstract}

Keywords: Concha, Feature Level Fusion, Multimodal Biometric System, Optic Disc, Unimodal Biometric System

\section{Introduction}

Now a day's most of the systems which needs controlled access to finance, networks, or any other critical security related applications prefer to have biometric identification systems which use physiological or behavioral traits to provide secured access to the system. The error rates associated with unimodal biometric system are quite high and this makes it unacceptable for deployment in security critical applications. To overcome the limitations faced with unimodal system multimodal systems were used. Multimodal systems are more reliable, due to the presence of multiple, a fairly independent piece of evidence. The main advantage of using multimodal system is spoofing becomes difficult for an intruder.

In this study, a new way of locating and extracting optic disc from retina is presented. Concha part of ear which is mostly free from occlusions is used as another biometric trait. Optic disc and concha, which are used as biometric traits here, are reliable and secured.

\section{Related Work and Motivation}

Retina's blood vessel pattern is one of the most stable and reliable biometric feature for person identification because manipulation of pattern is difficult. Karthikeyan and Rengarajan (2014) 
developed a glaucoma diagnosis system which classifies the retinal images based on the behavior of texture features as a function of gray level quantization. Kavitha and Duraiswamy (2011) proposed an automated system to extract blood vessels and detect exudates to screen diabetic retinopathy. Dehghani et al. (2012) presented a new approach for localising the optic disc and has sugessted that future research can be carried with optic disc for human recognition. A new approach to automatically locate and detect optic disc boundary was presented by (Siddalingaswamy and Prabhu, 2010). Later a new method for automatic detection of optic disc and other bright lesions, such as hard exudates and cotton wool spots in colour fundus images was presented by (Ahmed et al., 2011). Barkhoda et al. (2011) proposed an identification system based on retina image and this suggested system uses angular and radial partitioning for feature extraction. $\mathrm{Xu}$ et al. (2005) showed that the blood vessel skeleton can serve as biometric feature for fundus vessel image. Optic disc and fovea was localized using PCA by (Mudassar and Butt, 2013). Automated retinal optic disc segmentation using differential windowing in the polar coordinate domain was done by (Kavitha and Duraiswamy, 2013).

More research is done on identifying the person using ear as biometric trait compared to other techniques like face, eye, or fingerprint recognition. EAR has stable structure where its features are preserved well even at old age. Main advantage of using ear as biometric trait is it does not suffer from any changes when facial expression changes. Abaza et al. (2013) presented a systematic discussion which includes available databases, detection and feature extraction techniques, as well as a survey of some unsolved ear recognition problems. An American police officer (Iannarelli, 1989), proposed a first nonautomated ear recognition system based on a set of 12 measurements. Prior works on this include those by (Victor et al., 2002) on Principal Component Analysis of ear images and a further improvement of the same by (Chang et al., 2003). The geometric method of feature extraction from human ear images was proposed by (Choras, 2005). A very significant improvement was demonstrated by (Hurley et al., 2005) and he used force field feature extraction to map the ear to an energy field (2005). An ear detection method fusing range images and 2Dcolor images was developed by (Yan and Bowyer, 2007). The algorithm developed by them works by locating concha and then uses active contour to determine ear's outer boundary. A new feature extraction approach for ear identification using localized orientation was done by (Kumar and $\mathrm{Wu}, 2011$ ).

Based on the literature survey it is found that optic disc from is not used for identification. In this study, optic disc of retina is extracted by the new Region Of Interest (ROI) locator and concha part of the ear, taken as another biometric trait, is extracted by cropping. Different transforms DCT, Haar, force field are applied to the extracted features and the output is normalized. The normalized output of both features are fused and from the fused set statistical features like mean, median, standard deviation and kurtosis are extracted. These extracted statistical features are stored in the database. Euclidean distance measure is applied to identify the person. Then based on distance measure with the stored template match/no match are found. Performance analysis of this multimodal biometric recognition system is done using Match Rate (MR), False Match Rate (FMR), False Non Match Rate (FNMR) and Equal Error Rate (EER).

\section{Multimodal Biometric Recognition System}

A system that use multiple biometric characteristics is multimodal system. Emerging trend is using multimodal system for identification.

In our multimodal Biometric recognition system shown above in Fig. 1, the required Retinal input image is taken from the DRIVE and VARIA database and Ear image is taken from database provided by IIT Delhi provided by Hong Kong Polytechnic University. After acquiring input image pre-processing (Which means processing the image to suitable format) is done and Region Of Interest (ROI) is extracted. To the extracted ROI different transforms are applied, normalized and then fused. From the fused data statistical features like Mean, Median, Standard deviation and Kurtosis, are extracted and stored in database for enrollment. For identification same procedure is repeated and matching is done with the stored template using Euclidean distance measure.

Measures which are made with any biometric system are False Match Rate (FMR), False Non Match Rate (FNMR), Match Rate (MR) and Equal Error Rate (EER). The aim of multimodal biometric system is to reduce False Match Rate (Falsely identifying wrong person as right person).

False Non Match Rate (Falsely rejecting the right person as wrong person), Equal Error Rate (rate at which FMR and FNMR are equal) and increase Match Rate (Identifying the right person correctly).

\section{Anatomy of Eye}

A tough white covering called the sclera protects the eye. Part of the white sclera can be seen in the front of the eye. A clear, delicate membrane called the 
conjunctiva covers the sclera. At the front of the eye is the cornea. The cornea is the clear part of the eye's protective covering. It allows light to enter the eye. The iris is the colored part of the eye that contracts and expands so the pupil can let just the right amount of light into the eye. The light is directed by the pupil to the lens. The lens focuses the light onto the retina (lining the back of the eye). Nerve fibers in the retina carry images to the brain through the optic nerve. The Fig. 2 shown below shows the anatomy of Eye.

\section{The Optic Disc}

You have millions of nerve fibers that run from your retina to the optic nerve. These fibers meet at the optic disc. As fluid pressure within your eye increases, it damages these sensitive nerve fibers and they begin to die. As they die, the disc begins to hollow and develops a cupped or curved shape. If the pressure remains too high for too long, the extra pressure can damage the optic nerve and result in vision loss.

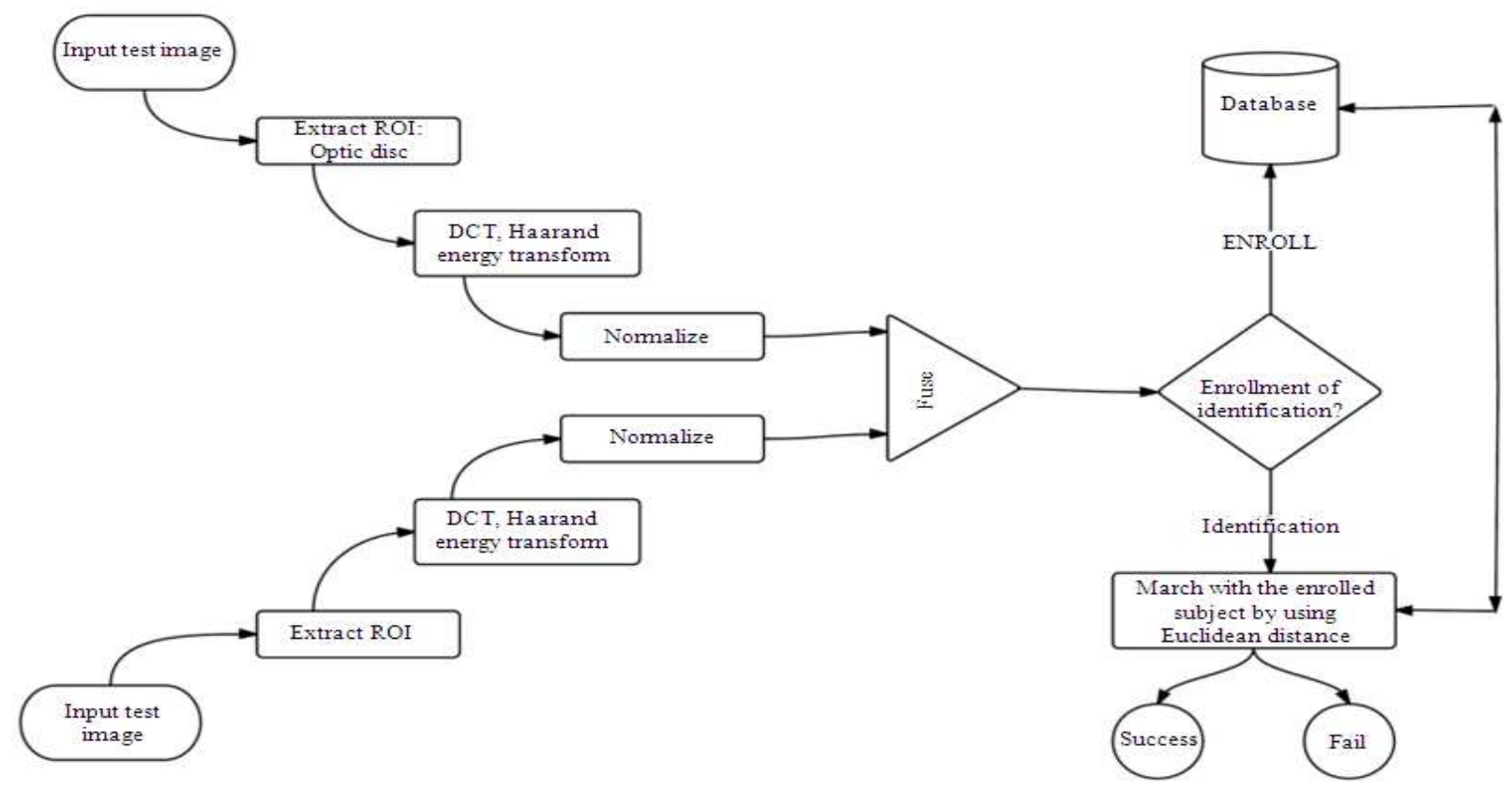

Fig. 1. Multimodal biometric recognition system

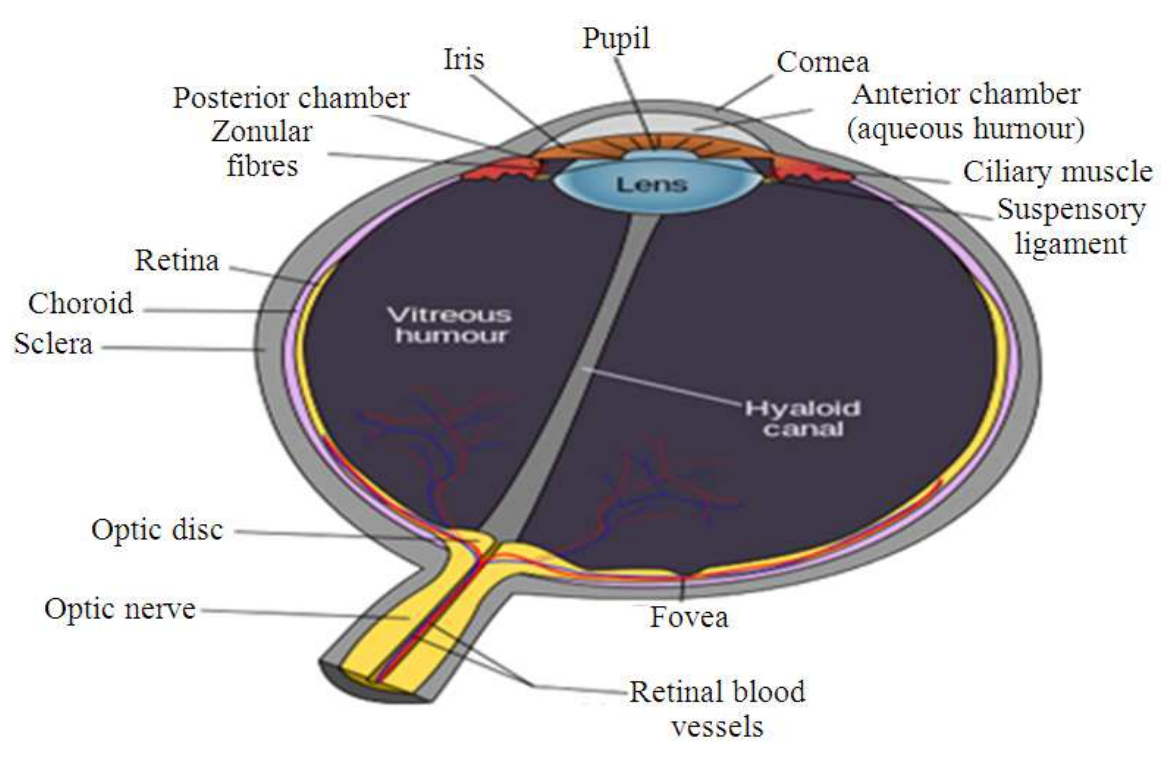

Fig. 2. Anatomy of eye 


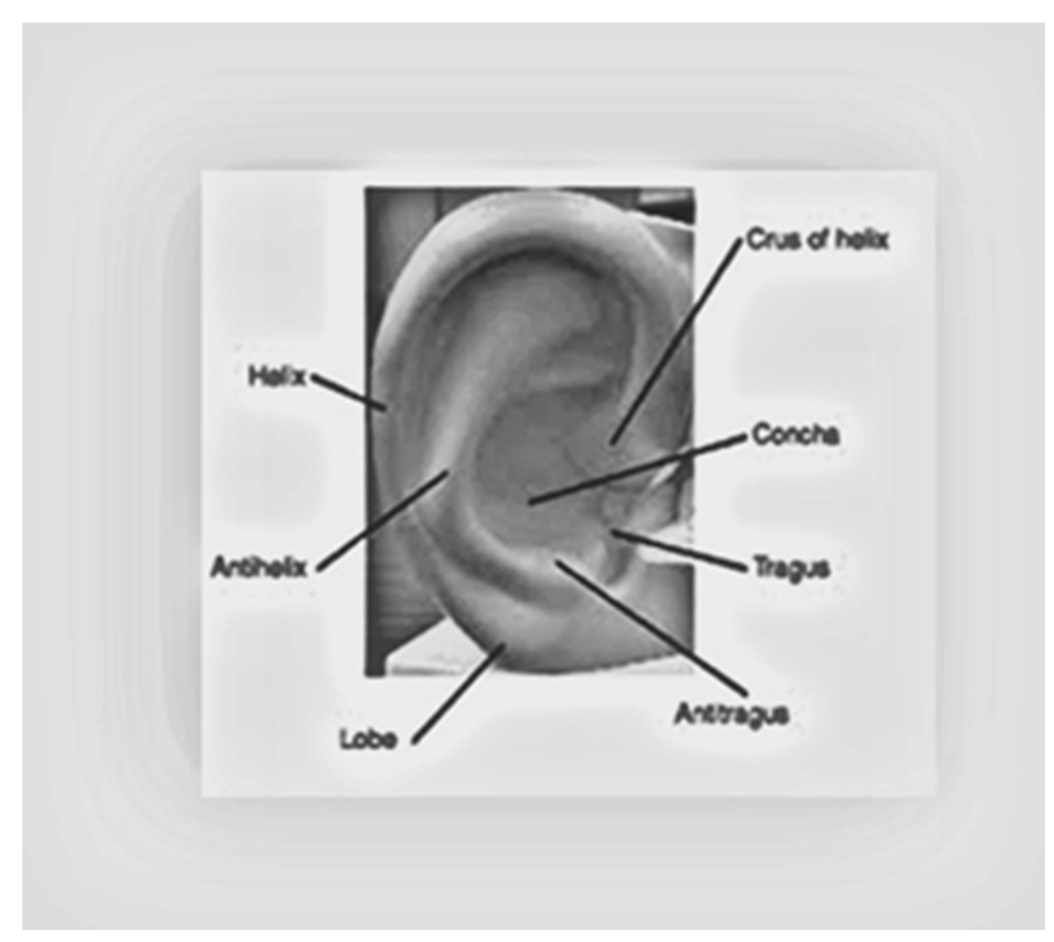

Fig. 3. Anatomy of ear

\section{Anatomy of Ear}

The parts of the ear are shown above in Fig. 3. The human ear has External ear: One of two organs of hearing on the sides of the head comprised of internal and external parts, Helix: Fold surrounding the auricle. Navicular fossa: Boat-shaped fold of the ear, Root of the helix: Part of the helix. Anthelix: Curved ridge in front of the helix, Tragus: Prominence over and in front of the acoustic meatus. Lobe: Fleshy part at the base of the ear, Antitragus: Ear projection opposite the tragus, External auditory meatus: Mouth of the canal of the temporal bone that carries sounds to the eardrum, Concha: Deep fossa of the external ear and Scapha: Hollow at the end of the helix.

\section{Materials and Methods}

The work is divided in to three phases.

First phase consists of preprocessing, second phase consists of feature extraction and third phase consists of matching. MATLAB R2010a is used for processing.

\section{First Phase (Pre-Processing)}

This is the very basic step in case of any biometric system. Here in this study the color image or gray image is converted to the binary image so, the Retinal image is taken form DRIVE and VARIA database.
DRIVE database has 40 images with a resolution of $565 \times 584$. This image is resized to $512 \times 512$ so that it will be in the powers of two. VARIA database has 233 images from 139 different individuals with a resolution of $584 \times 768$.

The Ear image taken from IIT Delhi Database has 125 different subjects and each subject has a minimum of three images. The resolution of each image is $272 \times 204$. This image is resized to meet the requirement.

\section{Second Phase (Feature Extraction) \\ Optic Disc from Retina}

To locate the optic disc of retinal blood vessels a new method ROI Locator is proposed and applied to the pre-processed image which returns Region Of Interest (ROI). This reduces the size of the image for further processing. ROI locator is based on locating the maximum white pixels in a given window of size $64 \times 64$. Moving the window over the image, pixel by pixel through the whole image of retina a window having maximum number of white pixels is obtained. The resultant window having maximum number of white pixels generated by ROI Locator is the actual region of interest. Figure $4 \mathrm{a}$ shown below isRetinal image and Fig. $4 \mathrm{~b}$ shown below is the optic disc extracted by the ROI Locator. 


\section{Concha Part}

The depression encircled by the anti tragus, the curving part of the anti helix and the inferior anti helix crus. It consists of two parts: Superior concha and inferior concha. Here concha part of the ear is extracted by cropping. After extraction of concha part from the ear image, it is resized to the size of extracted ROI of Retina which is of size $64 \times 64$. Figure 5 a shown below is the Ear image and Fig. 5b shown below is the Concha part of ear extracted by Cropping

\section{Transformation}

The principle advantage of image transformation is the removal of redundancy between neighboring pixels. This leads to uncorrelated transform coefficients which can be encoded independently.

\section{Discrete Cosine Transform}

A technique for converting signal into elementary frequency components. 1D DCT is applied to each row of $\mathrm{F}$ and then to each column of the result. The general equation for a $2 \mathrm{D}(\mathrm{N}$ by $\mathrm{N}$ image) DCT is defined by the following Equation 1:

$$
\begin{aligned}
& F(u, v)=\frac{2}{N} C(u) C(v) \\
& \sum_{y=0}^{N-1} \sum_{x=0}^{N-1} f(x, y) \cos \left[\frac{(2 x+1) u \pi}{2 N}\right] \cos \left[\frac{(2 y+1) v \pi}{2 N}\right]
\end{aligned}
$$

\section{Haar Transform}

The Haar transform is one of the simplest and basic transformations from the space/time domain to a local frequency domain, which reveals the space/timevariant spectrum. The Haar transform was proposed in 1910 by a Hungarian mathematician Alfred Haar. The attracting features of the Haar transform are its ability to analyses the local feature. The Haar transform $H T^{n}$ $(f)$ of an $\mathrm{N}$-input function $X^{n}(f)$ is the $2^{\mathrm{n}}$ element vector as given by the following Equation 2:

$$
H T^{n}(f)=H^{n} T^{n}(f)
$$

\section{Force Field Transform (Hurley et al., 2005)}

The image is transformed by treating the pixels as an array of $\mathrm{N}$ mutually attracting particles that act as the source of a Gaussian force field. In a similar way to Newton's Law of Universal Gravitation, the pixels are considered to attract each other according to the product of their intensities and inversely to the square of the distances between them. Each pixel is assumed to generate a spherically symmetrical force field so that the force $F_{i}\left(r_{j}\right)$ exerted on a pixel of unit intensity at the pixel location with position vector $r j$ by a remote pixel with position vector $r i$ and pixel intensity $P\left(r_{i}\right)$ is given by the following Equation 3:

$$
F_{i}\left(r_{j}\right)=P\left(r_{j}\right)\left(\frac{\left(r_{i}-r_{j}\right)}{\left(r_{i}-r_{j}\right)^{3}}\right.
$$

Total force $F\left(r_{j}\right)$ exerted on a pixel of unit intensity at the pixel location with position vector $r_{j}$ is the vector sum of all the forces due to the other pixels in the image and is given by Equation 4:

$$
F_{i}\left(r_{j}\right)=\sum_{i=0}^{N-1} 1 P\left(r_{j}\right)\left(\frac{\left(r_{i}-r_{j}\right)}{\left(r_{i}-r_{j}\right)^{3}}\right.
$$

\section{Transformation Applied to Optic Disc of Retina}

When DCT is applied to optic disc it transforms the image from spatial domain to frequency domain. The optic disc is given as input image to DCT function so as to transform it for further processing. In the above Fig. 6a shows optic disc after DCT Transform When Haar transform is applied to extract optic disc it gives the Haar transformed image as shown in Fig. 6b. According to force field transform each pixel is treated as point mass and distance between two nearest pixels as unity. On applying Newton's classical theory of gravitation energy of the selected ROI of the image is obtained as shown in Fig. 6c.

After extraction of concha part from the ear image, it is resized to the size of extracted ROI of Retina which is of size $64 \times 64$. The Fig. $7 \mathrm{a}$ shown is the DCT transformed concha image. Figure $7 b$ is the Hear transformed concha image and Fig. $7 \mathrm{c}$ is the Force field transformed concha image.

\section{Normalization of Output After Transformations}

As the output of Energy Transform, Hear and DCT functions are different, it is to be normalized. We can achieve both a speedup in processing and an improvement in image quality by normalizing the transformed output Math 572).

\section{Fusion of the Extracted Features}

The normalized output of optic disc extracted by ROI locator and concha extracted by cropping are added together to get fused template. This fused data is stored in Database. 


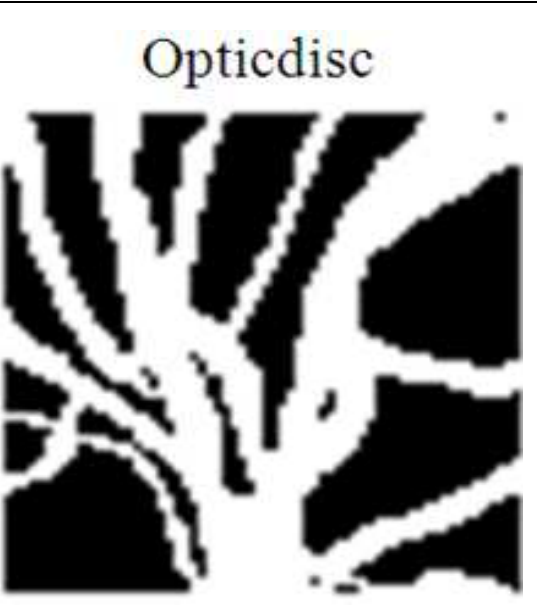

(b)

Fig. 4. (a) Retinal image and (b) Optic disc extracted by the ROI locator

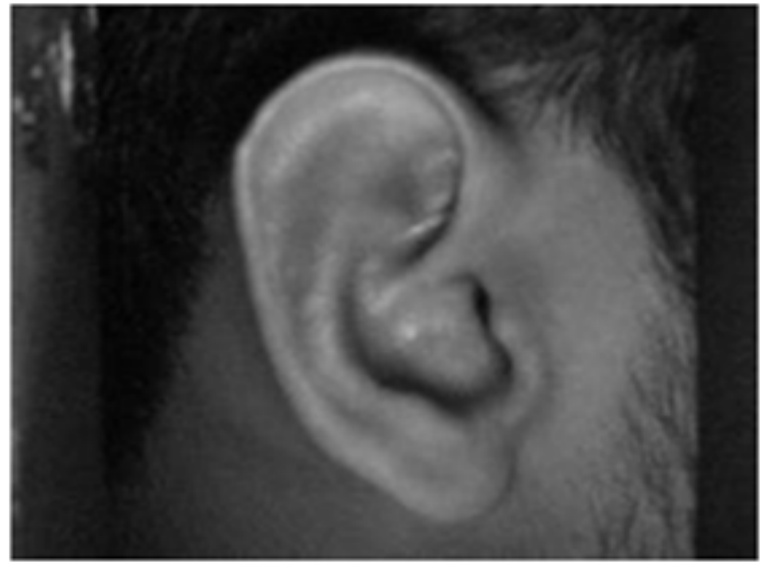

(a)

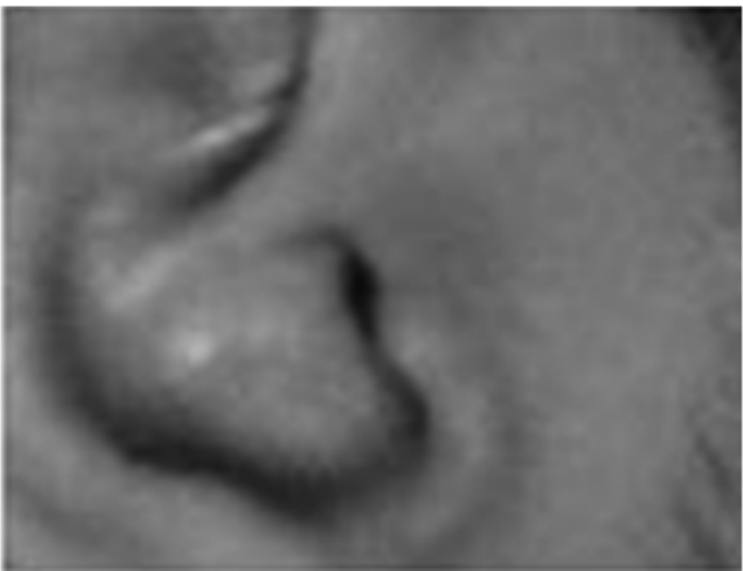

(b)

Fig. 5. (a) Ear image and (b) Concha extracted by cropping

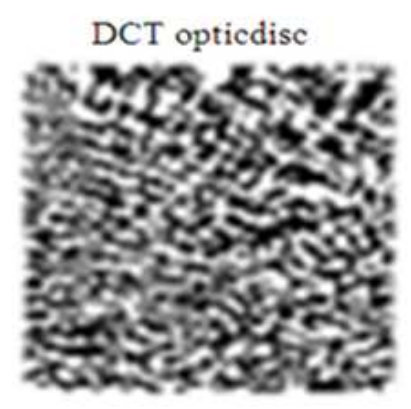

(a)

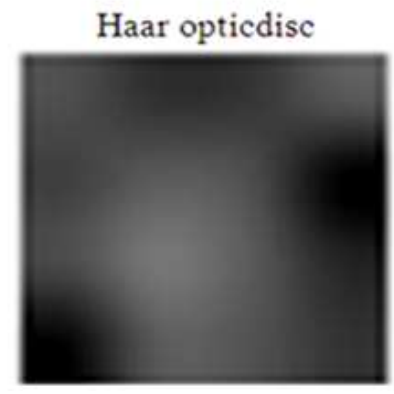

(b)

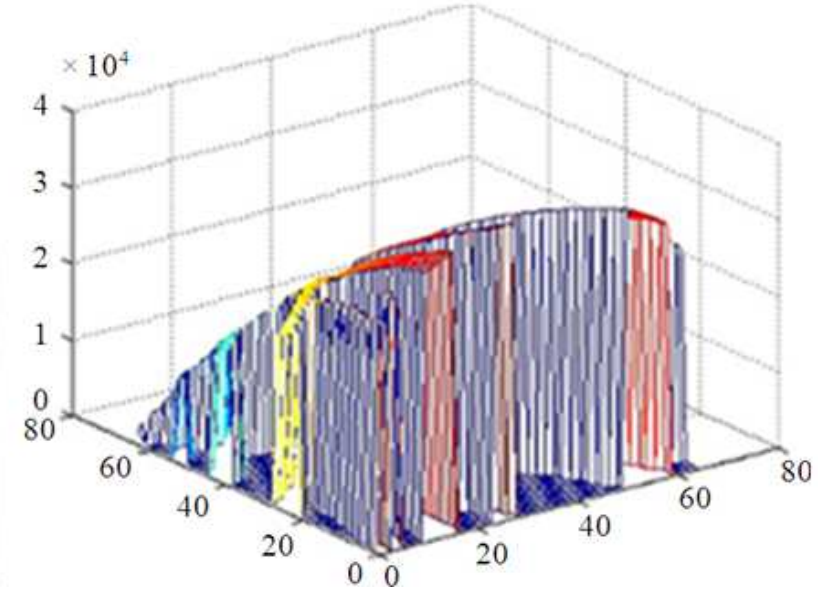

(c)

Fig. 6. (a) DCT applied to optic disc (b) haar applied to optic disc (c) force field applied to optic disc 


\section{Third Phase (Matching by Euclidean Distance Method)}

This method calculates the distance between the training and testing images. The test image matches with the trained image when the distance between them is small when compared with other distances. The Euclidean Distance (ED) is calculated as given in Equation 5:

$$
\begin{aligned}
& \text { fori }=1: \text { EuclidDist }(i)= \\
& \operatorname{sqrt}\left(\operatorname{sum}\left((\text { BioMetricFeat }(i,:) \backslash-\text { Feat }){ }^{2}\right)\right) ; \text { End }
\end{aligned}
$$

where, Biometric feat represents training pixels and Feat indicates the testing pixels in the training and testing database. It was tested with the datasets of DRIVE, VARIA and IIT Delhi.

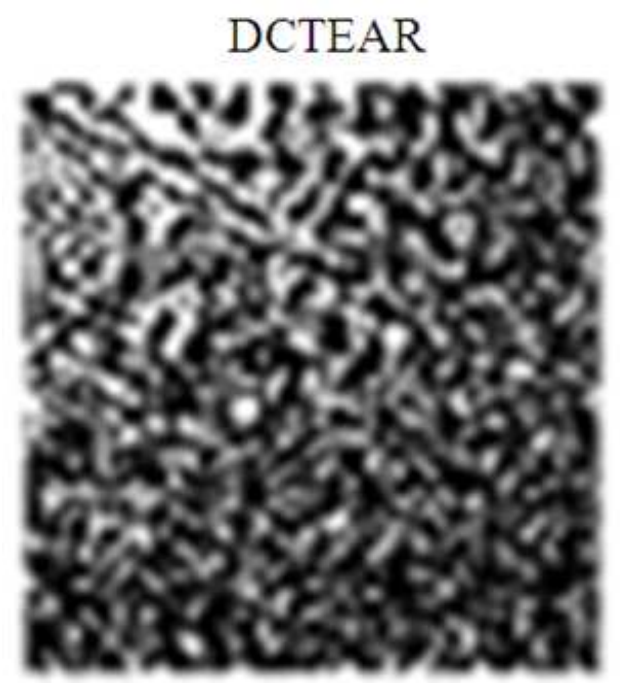

(a)

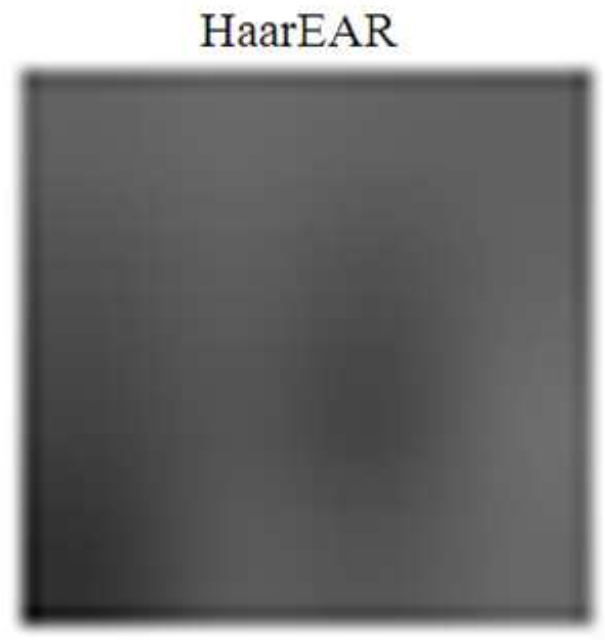

(b)

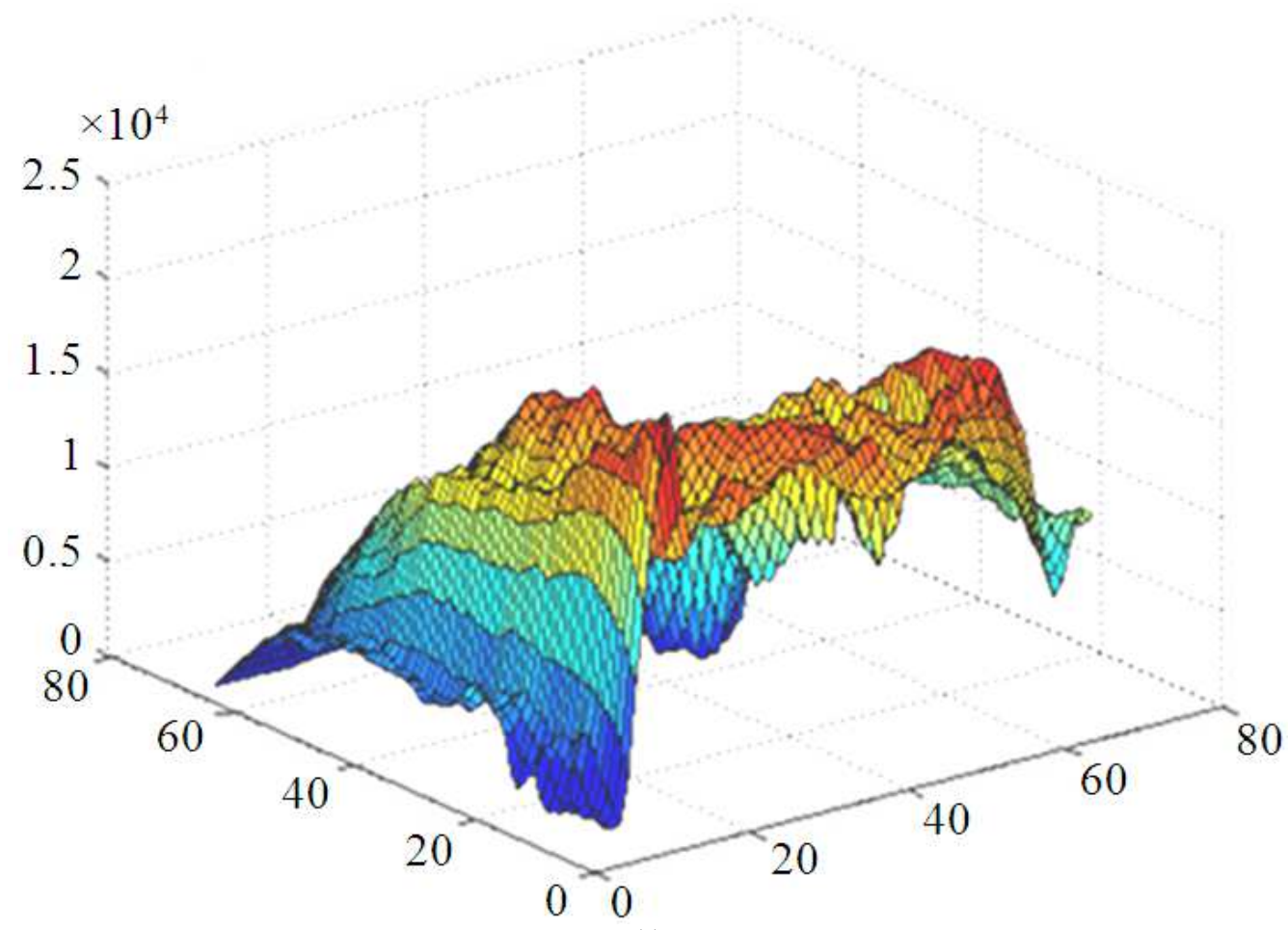

(c)

Fig. 7. (a) DCT applied to concha (b) haar applied to concha (c) force field transformed concha image 
The pseudo code for multimodal biometrics taking optic disc of retina and concha of ear is given below.

Pseudo Code for Multimodal Biometrics for Identification

\section{Input: Retina image and Ear Image}

\section{Output: Match/No Match:}

- Take Input Test images

- Select Region Of Interest (ROI) from Retina by new method ROI Locator to extract optic disc

- Select Region Of Interest (ROI) from ear image by cropping

- Apply DCT, Haar and Energy Transform function to the extracted portion, optic disc of the Retinal image

- Apply DCT, haar and energy transform function to the extracted portion, concha of the ear image

- Normalize the output of previous two operations

- Fuse the normalized features

- Extract statistical features like mean ,median, standard deviation and Kurtosis from the fused features and store it in the database

- Match with the enrolled subject based on Euclidean distance measure

\section{Results}

The Retina images and Ear images is taken as the biometric traits in this Multimodal biometric recognition system. The retina images with 40 subjects consisting of both right and left eye were taken for analysis. Ear database taken for analysis here consists of 40 subjects with each subject having 3 minimum and 6 maximum images in each subject with different angle, illumination and occlusions. Three images from each subject are used for enrolment and six mages of each subject is used for testing. Match Rate (MR), False Match Rate (FMR), False Non Match Rate (FNMR) Equal Error Rate (EER) are found for the extracted optic disc and concha using Euclidean distance method. Both the databases of retina (DRIVE and VARIA) was fused separately with IIT Delhi ear database and tested for person identification. The Match Rate (MR) was calculated against various thresholds for both retinal databases with ear database using Euclidean method.

\section{Discussion}

Zero false recognition,38 false rejection and $0.19 \%$ recognition rejection was observed with 200 fundus blood vessel images when cross comparisons experiment was carried out by (Xu et al., 2005). Similarity between different fundus blood vessel skeletons were measured and $90 \%$ matching rate was obtained with the database having fundus blood vessel images of 200 people where for each people 10 images were taken at different time. Experimentation was conducted with two databases in our paper and with optic disc being extracted we were able to achieve more than $95 \%$ matching rate.

The Table 1 and 2 shown below gives the Match Rate for Multimodal Biometric System with various thresholds for DRIVE and VARIA database. When the threshold is above 7 Match Rate (MR) is above $95 \%$.

The Fig. 8a-d shown is the Match rate with and without ROI for DRIVE and VARIA database. The Match Rate is plotted for various thresholds.

In the Fig. $8 \mathrm{~b}$ and $\mathrm{d}$ MRDCT shows the result when DCT is applied to both retina and ear, MR Haar shows the result when Haar is applied to both retina and ear, MR Energy shows the result when Force field transform is applied to both retina and ear, MRDCT Energy shows the result when DCT is applied toretina and Force field transform is applied to ear and MR Haar Energy shows the result when Haar is applied to retina and Force field transform is applied to ear. The same notation is followed in case of with ROI that is for optic disc of retina and concha of ear which is shown in Fig. 8a and c.

Figure 9 shows the Match time for different transformation with Extraction of ROI and without extraction of ROI.

Matching Time with ROI locator and Without ROI locator is shown in Table 3 and Fig. 9. It is calculated for both DRIVE and VARIA database.

\section{Storage Space and Processing Time}

The storage space occupied by retina image is 12 $\mathrm{KB}$ for DRIVE database and $440 \mathrm{~KB}$ for VARIA database. By the extraction of ROI the storage space occupied by optic disc is $4 \mathrm{~KB}$ for DRIVE database and $8 \mathrm{~KB}$ for VARIA database. As the space occupied by optic disc and the processing Time is very less compared to retinal image this method will speed up the identification time.

\section{Equal Error Rate}

The Fig. 10a and $\mathrm{b}$ shown above gives Equal error rate for DRIVE and VARIA database and it is around $5 \%$ for DRIVE database and less than $12 \%$ for VARIA database when DCT transform is applied to optic disc and Energy transform is applied to Ear. Lower the equal error rate value, higher is the accuracy of the biometric system. 


\section{Results 2}

\section{Unimodal System}

The new algorithm developed to extract optic disc from retinal image is analyses and tested separately with and without the ROI locator for unimodal system.

\section{Discussion (Unimodal)}

The experimental results for Unimodal system is shown in Fig. 11, Table 4 and 5. In this table Opt Haar, Opt Energy, Opt DCT shows the Match Rate with ROI locator and Ret Haar, Ret Energy, Ret DCT shows the Match rate without ROI locator.

Table 1. Match rate with and without ROI for drive database

\begin{tabular}{lcrrrrrrrrrrrr}
\hline Threshold & \multicolumn{1}{c}{7} & \multicolumn{1}{c}{7.25} & \multicolumn{1}{c}{7.5} & \multicolumn{1}{c}{7.75} & \multicolumn{1}{c}{8} & \multicolumn{1}{c}{8.25} & \multicolumn{1}{c}{8.5} & \multicolumn{1}{c}{8.75} & 9 & \multicolumn{1}{c}{9.25} & \multicolumn{1}{c}{9.5} & \multicolumn{1}{c}{9.75} & \multicolumn{1}{c}{10} \\
\hline Haar_WithROI & 92.6 & 92.6 & 94.3 & 95.7 & 95.7 & 95.7 & 95.7 & 95.7 & 95.70 & 95.7 & 95.70 & 97.10 & 97.10 \\
Energy_WithROI & 97.1 & 97.1 & 97.1 & 97.1 & 97.1 & 97.1 & 98.5 & 98.5 & 98.50 & 98.5 & 98.50 & 98.50 & 98.50 \\
DCT_WithROI & 20.0 & 0.2 & 18.3 & 18.3 & 18.3 & 16.6 & 16.6 & 16.6 & 16.60 & 16.6 & 0.15 & 0.15 & 0.15 \\
DCT_Energy_WithROI & 95.7 & 95.7 & 97.1 & 97.1 & 97.1 & 98.5 & 100.0 & 100.0 & 100.00 & 100.0 & 100.00 & 100.00 & 100.00 \\
Haar_Energy_WithROI & 95.6 & 95.6 & 95.6 & 97.1 & 97.1 & 97.1 & 97.1 & 97.1 & 98.50 & 98.5 & 98.50 & 98.50 & 98.50 \\
Haar_WO_ROI & 95.7 & 95.7 & 95.7 & 95.7 & 97.1 & 97.1 & 97.1 & 97.1 & 97.10 & 97.1 & 97.10 & 97.18 & 97.18 \\
Energy_WO_ROI & 98.5 & 100.0 & 100.0 & 100.0 & 100.0 & 100.0 & 100.0 & 100.0 & 100.00 & 100.0 & 100.00 & 100.00 & 100.00 \\
DCT_WOROI & 43.3 & 43.3 & 43.3 & 41.7 & 38.3 & 38.3 & 38.3 & 36.6 & 0.35 & 33.3 & 33.30 & 33.30 & 30.00 \\
DCT_Energy_WOROI & 95.7 & 95.7 & 95.7 & 95.7 & 95.7 & 95.7 & 95.7 & 95.7 & 97.10 & 97.1 & 97.10 & 97.10 & 97.10 \\
Haar_Energy_WOROI & 94.2 & 94.2 & 94.2 & 94.2 & 94.2 & 94.2 & 94.2 & 95.6 & 95.60 & 95.6 & 95.60 & 95.60 & 95.60 \\
\hline
\end{tabular}

Table 2. Match rate with and without ROI for VARIA database

\begin{tabular}{|c|c|c|c|c|c|c|c|c|c|c|c|c|c|}
\hline Thres & 7 & 7.25 & 7.5 & 7.75 & 8 & 8.25 & 8.5 & 8.75 & 9 & 9.25 & 9.5 & 9.75 & 10 \\
\hline aa & 9 & 92.9 & & & & & 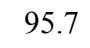 & & 7 & 7 & 1 & & 5.7 \\
\hline KU1 & .5 & 98.5 & & & & & 98.5 & & 98.5 & 98.5 & 98.5 & 98.5 & 98.5 \\
\hline & 98.5 & & & & & & & & & & & & 100.0 \\
\hline OI & 100.0 & 100 & 10 & & & & & & & & & & 100.0 \\
\hline 1 & 95.7 & 0 & 7 & 7 & 7 & 7 & .7 & 7 & 95.7 & 95.7 & 95.7 & .7 & 95.7 \\
\hline _ROI & .5 & 98.5 & 5 & 8.5 & 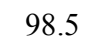 & 5 & 8.5 & 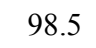 & 98 & 98.5 & 5 & 5 & 98.5 \\
\hline CT_WOROI & 35.0 & 31 & 26.6 & & & & 18.3 & & 16.6 & 16.6 & 15.0 & 11.6 & 10.0 \\
\hline DCT_Energy_V & 5.7 & & & & & & 95.7 & & 95.7 & 95.7 & & 95.7 & 95.7 \\
\hline Haar_Energy_WOROI & 92.7 & 92.7 & 92.7 & 92.7 & 92.7 & 92.7 & 92.7 & 94.1 & 94.1 & 94.1 & 94.1 & 95.6 & 95.6 \\
\hline
\end{tabular}

Table 3. Match time for DRIVE and VARIA database

\begin{tabular}{lllll}
\hline Transformations & $\begin{array}{l}\text { Match Time with ROI } \\
\text { in seconds (DRIVE) }\end{array}$ & $\begin{array}{l}\text { Match Time without } \\
\text { ROI in seconds (DRIVE) }\end{array}$ & $\begin{array}{l}\text { Match Time without ROI } \\
\text { in seconds (VARIA) }\end{array}$ & $\begin{array}{l}\text { Match Time with ROI } \\
\text { in seconds (VARIA) }\end{array}$ \\
\hline HAAR & 0.0267 & 0.0267 & 4.32 & 5.48 \\
Energy & 0.0257 & 0.0261 & 6.22 & 6.21 \\
DCT & 0.0262 & 0.0279 & 5.06 & 5.79 \\
HAAR_Energy & 0.0256 & 0.0260 & 5.61 & 6.55 \\
DCT_Energy & 0.0260 & 0.0265 & 5.96 & 6.98 \\
\hline
\end{tabular}

Table 4. Unimodal system with and Without ROI for DRIVE database

\begin{tabular}{llllllllllllll}
\hline Threshold & 7 & 7.25 & 7.5 & 7.75 & 8 & 8.25 & 8.5 & 8.75 & 9 & 9.25 & 9.5 & 9.75 & 10 \\
\hline Opt Haar & 100 & 100 & 100 & 100 & 100 & 100 & 100 & 100 & 100 & 100 & 100 & 100 & 100 \\
Opt Energy & 100 & 100 & 100 & 100 & 100 & 100 & 100 & 100 & 100 & 100 & 100 & 100 & 100 \\
Opt DCT & 100 & 100 & 100 & 100 & 100 & 100 & 100 & 100 & 100 & 100 & 100 & 100 & 100 \\
Ret Haar & 100 & 100 & 100 & 100 & 100 & 100 & 100 & 100 & 100 & 100 & 100 & 100 & 100 \\
Ret Energy & 100 & 100 & 100 & 100 & 100 & 100 & 100 & 100 & 100 & 100 & 100 & 100 & 100 \\
Ret DCT & 100 & 100 & 100 & 100 & 100 & 100 & 100 & 100 & 100 & 100 & 100 & 100 & 100 \\
\hline
\end{tabular}


Table 5. Unimodal system with and without ROI for VARIA database

\begin{tabular}{|c|c|c|c|c|c|c|c|c|c|c|c|c|c|}
\hline Threshold & 7 & 7.25 & 7.5 & 7.75 & 8 & 8.25 & 8.5 & 8.75 & 9 & 9.25 & 9.5 & 9.75 & 10 \\
\hline OptHaar & 100 & 100 & 100 & 100 & 100 & 100 & 100 & 100 & 100 & 100 & 100 & 100 & 100 \\
\hline Opt Energy & 100 & 100 & 100 & 100 & 100 & 100 & 100 & 100 & 100 & 100 & 100 & 100 & 100 \\
\hline Opt DCT & 100 & 100 & 100 & 100 & 100 & 100 & 100 & 100 & 100 & 100 & 100 & 100 & 100 \\
\hline Ret Haar & 100 & 100 & 100 & 100 & 100 & 100 & 100 & 100 & 100 & 100 & 100 & 100 & 100 \\
\hline Ret Energy & 100 & 100 & 100 & 100 & 100 & 100 & 100 & 100 & 100 & 100 & 100 & 100 & 100 \\
\hline Ret DCT & 100 & 100 & 100 & 100 & 100 & 100 & 100 & 100 & 100 & 100 & 100 & 100 & 100 \\
\hline
\end{tabular}

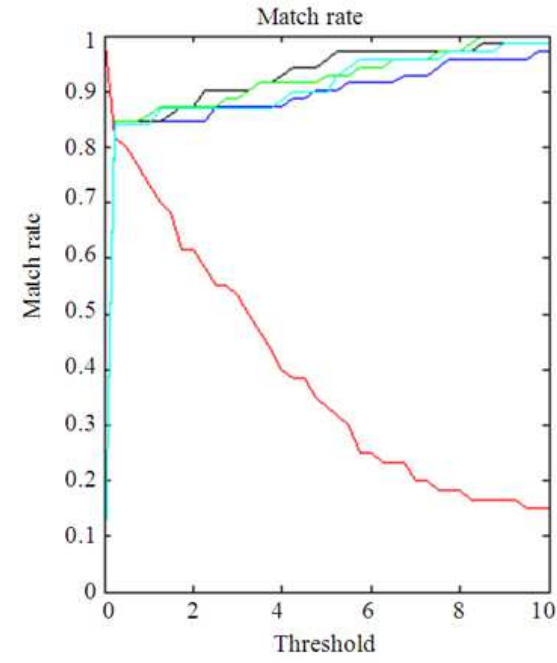

(a)

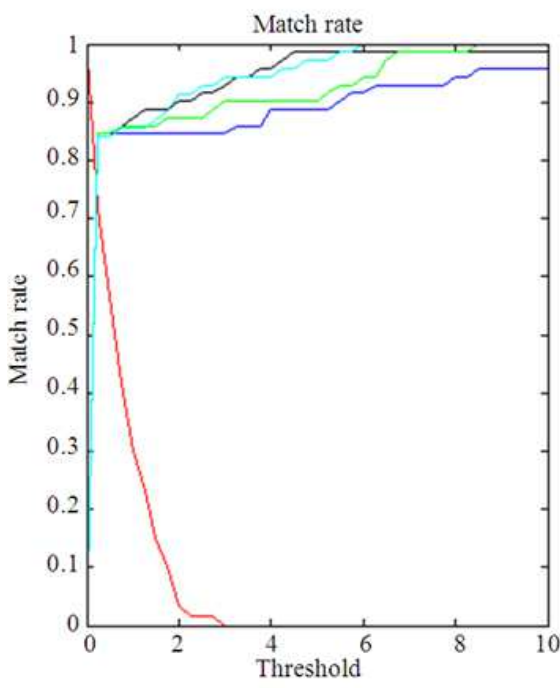

(c)

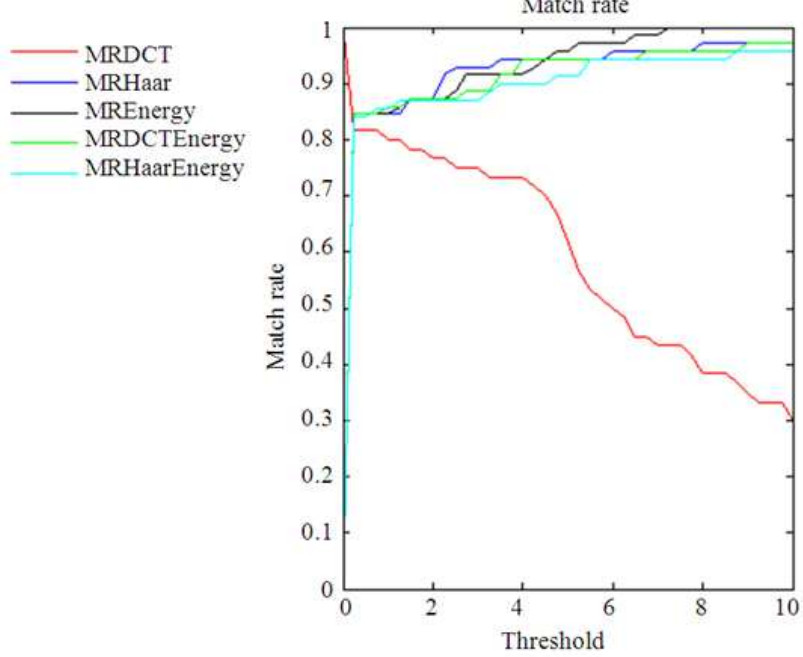

(b)
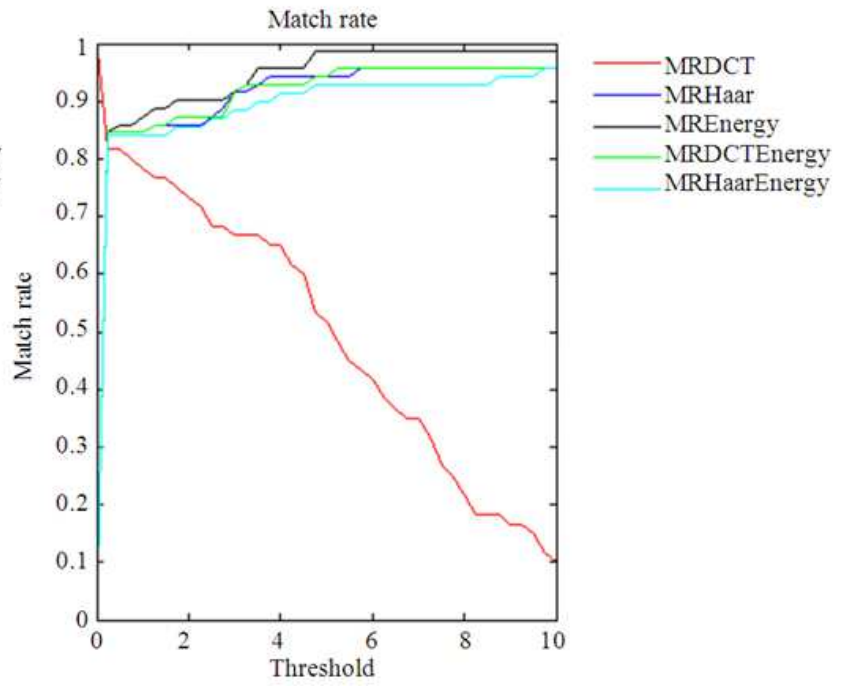

(d)

Fig. 8. (a) Match rate with ROI, (b) Without ROI for DRIVE database (c) Match rate with ROI and (d) Without ROI for VARIA database

It is observed that when threshold is varied from 0.25 to 10 with 0.25 differences, a matching rate of $100 \%$ is achieved with all the transforms applied to the system. With this new method of Extraction using ROI Locator excellent matching rate is obtained. 


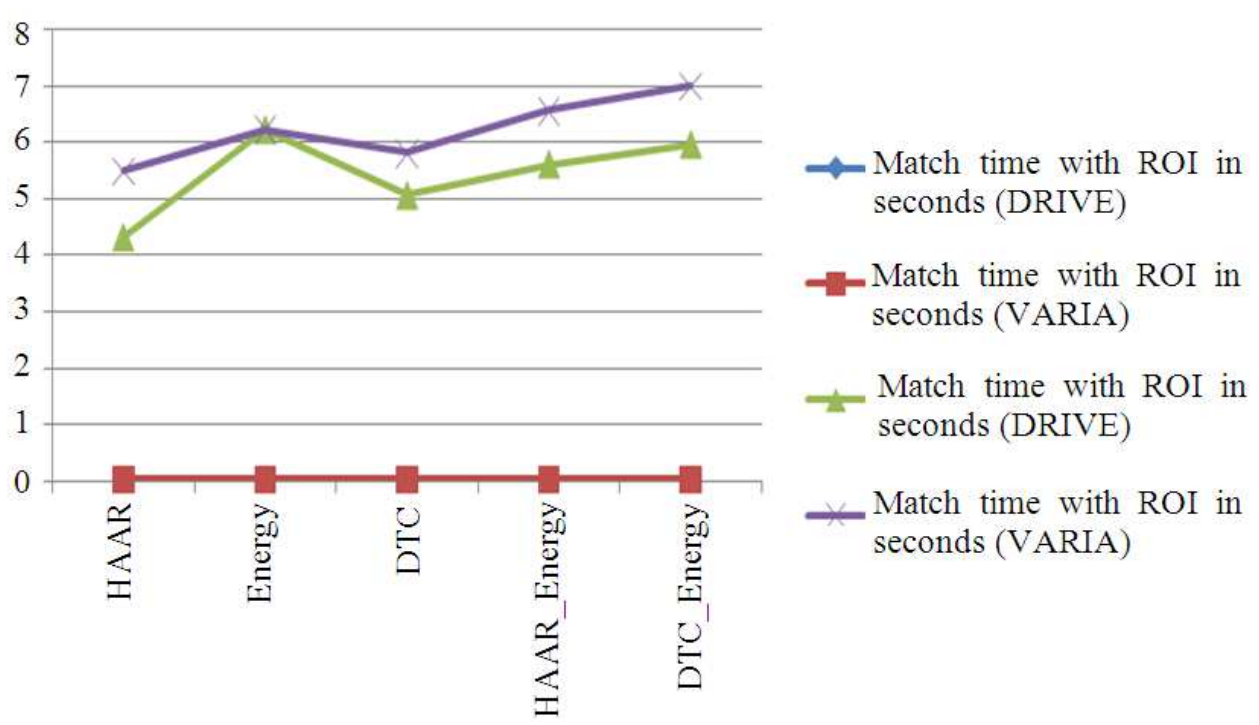

Fig. 9. Match time with and without ROI for different transformations

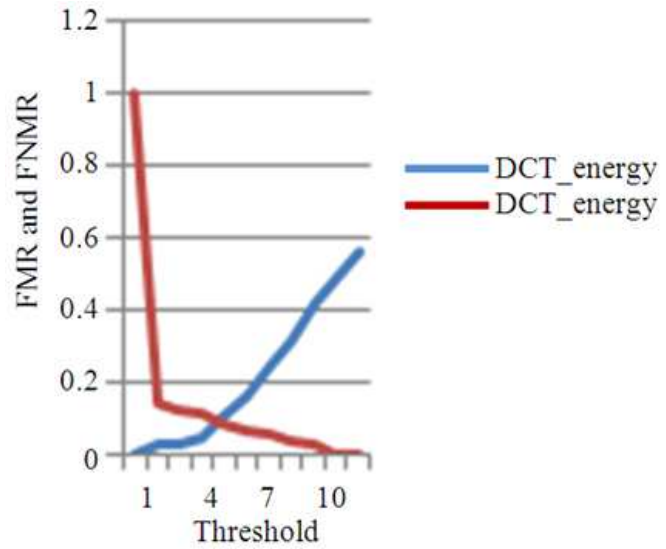

(a)

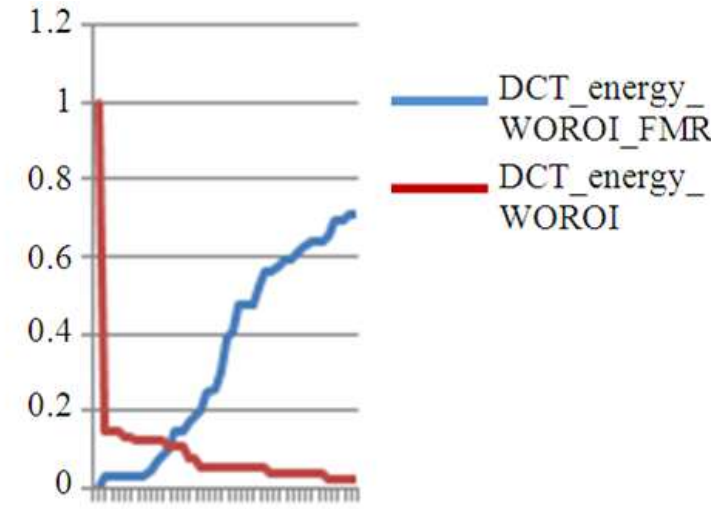

1815222936

(b)

Fig. 10. (a) Equal error rate for DRIVE and (b) equal error rate for VARIA database

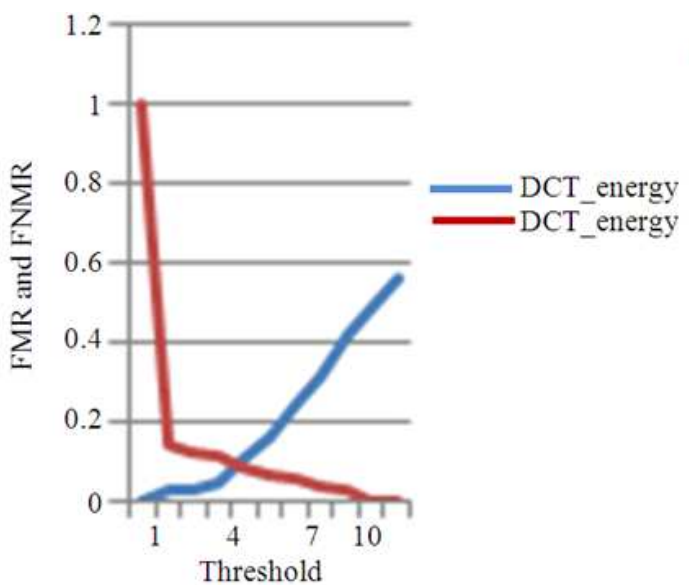

(a)

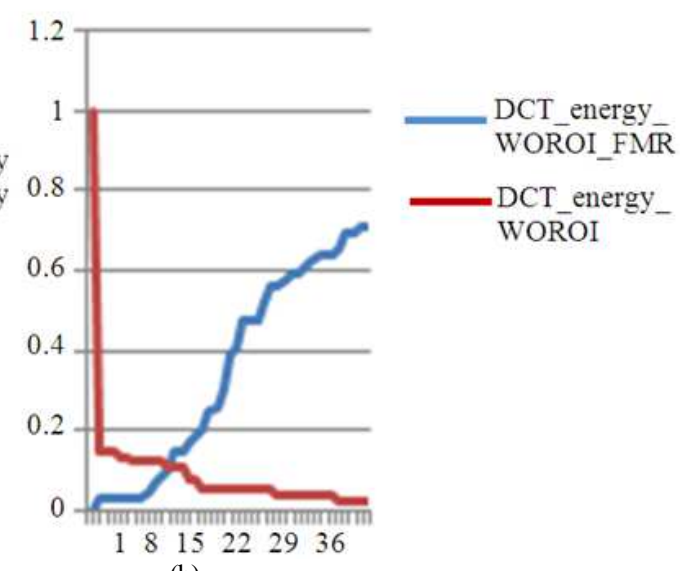

(b)

Fig. 11. (a) Shown above is the Matching Rate (MR) for unimodal system with Retina and optic disc 


\section{Conclusion}

In this study, we have presented a new method for extracting optic disc from Retina. It was observed that by applying different transforms to this new method of extraction the combination of DCT applied to optic disc and force field transform applied to ear gives good matching rate. The new method of extraction of optic disc using ROI Locator was implemented and tested with DRIVE and VARIA database. Unimodal system with ROI locator and without ROI locator was implemented and was observed that with both the databases the system was able to give $100 \%$ Matching Rate. Based on these results it was applied for multimodal system.

The performance of the system was analyzed by calculating FMR, MR, FNMR, EER, matching time and storage space. The matching time and storage space with ROI is less when compared to without ROI. Hence we conclude that when optic disc is used for person identification better results can be achieved.

The future scope of the work in this study would be to analyze the multimodal system with other transforms, Also the ear images can be tested with occlusions in concha part. The system can be tested with different levels of fusion and matching .Further study can be made to automatically extract concha part of the ear.

\section{Acknowledgement}

Our sincere thanks to Image sciences Institute for providing us with DRIVE database.

Research section, Digital Retinal Image for Vessel Extraction (DRIVE) Database, (2009). Utrecht, the Netherlands, Univ. Med. Center Utrecht, Image Sci. Institute.

http://www.isi.uu.nl/Research/Databases/DRIVE.

Our sincere thanks to the VARIA database which has a set of retinal images used for authentication purposes. The database includes 233 images, from 139 different individuals. The images have been acquired with a Top Con non-mydriatic camera NW-100 model and are optic disc centered with a resolution of $768 \times 584$.

Our sincere thanks to Mr. Ajay Kumar for supporting us with IIT Delhi Ear Database (Version1.0). (http://www4.comp.polyu.edu.hk/csajaykr/myhome/da tabase_request/ear/).

\section{Author's Contributions}

All authors equally contributed in this work.

\section{Ethics}

This article is original and contains unpublished material. The corresponding author confirms that all of the other authors have read and approved the manuscript and no ethical issues involved.

\section{References}

Abaza, A., R. Arun, H. Christina, Harrison, M.A.F and N. Mark, 2013. A survey on ear biometrics. ACM Comput. Surveys, 45: 1-22. DOI: $10.1145 / 2431211.2431221$

Ahmed, W.R., C. Eswaran and K. Dimyati, 2011. Diagnosis of diabetic retinopathy: Automatic extraction of optic disc and exudates from retinal images using marker-controlled watershed. J. M. Syst., 35: 1491-501. DOI: 10.1007/s10916-009-9426-y

Barkhoda, W., F. Akhlaqian, M.D. Amiri and M.S. Nouroozzadeh, 2011. Retina identification based on the pattern of blood vessels using fuzzy logic. EURASIP J. Adv. Signal Proc., 2011: 113113. DOI: $10.1186 / 1687-6180-2011-113$

Chang, K., K.W. Bowyer, S. Sarkar and B. Victor, 2003. Comparison and combination of ear and face images in appearance-based biometrics. IEEE Trans. Patt. Anal. Machine Int., 25: 1160-1165. DOI: 10.1109/TPAMI.2003.1227990

Choras, M., 2005. Ear biometrics based on geometrical feature extraction. Electron. Lett. Comput. Vis. Image Anal., 5: 84-95.

Dehghani, A., H.A. Moghaddam and M.S. Moin, 2012. Optic disc localization in retinal images using histogram matching. EURASIP. J. Image Video Proc. DOI: 10.1186/1687-5281-2012-19

Hurley, D.J., M.S. Nixon and J.N. Carter, 2005. Force field feature extraction for ear biometrics. Comput. Vision Image Understand., 98: 491-512. DOI: 10.1016/j.cviu.2004.11.001

Iannarelli, A., 1989. Ear Identification Paramount Publishing Company. 1st Edn., Freemont, California.

Karthikeyan, S. and N. Rengarajan, 2014. Performance analysis of gray level co-occurrence matrix texture features for glaucoma diagnosis. Am. J. Applied Sci., 11: 248-257. DOI: 10.3844/ajassp.2014.248.257

Kavitha, S. and K. Duraiswamy, 2011. Adaptive neuro-fuzzy inference system approach for the automatic screening of diabetic retinopathy in fundus images. J. Comput. Sci., 7: 1020-1026. DOI: $10.3844 /$ jcssp.2011.1020.1026

Kavitha, S. and K. Duraiswamy, 2013. An automatic retinal optic disc segmentation algorithm using differential windowing in the polar coordinate domain. Aus. J. Elec. Elect. Eng., 10: 33-34. DOI: 10.7158/E11-012.2013.10.1

Kumar, A. and C. Wu, 2011. Automated human identification using ear imaging. Patt. Recog., 45: 956-968. DOI: 10.1016/j.patcog.2011.06.005 
Mudassar, A.A. and S. Butt, 2013. Application of principal component analysis in automatic localization of optic disc and fovea in retinal images. J. Med. Eng., DOI: 10.1155/2013/989712

Siddalingaswamy, P.C. and G.K. Prabhu, 2010. Automatic localization and boundary detection of optic disc using implicit active contours. Int. J. Comput. Applic., 1: 1-5. DOI: 10.5120/171-298

Victor, B., K. Bowyer and S. Sarkar, 2002. An evaluation of face and ear biometrics. Proceedings of the 16th International Conference on Pattern Recognition, Aug. 11-15, IEEE Xplore Press, pp: 429-432. DOI: 10.1109/ICPR.2002.1044746
Xu, Z.W., X.X. Guo, X.Y. Hu and X. Cheng, 2005. The blood vessel recognition of ocular fundus. Proceedings of the International Conference on Machine Learning and Cybernetics, Aug. 18-21, IEEE Xplore Press, Guangzhou, China, 4493-4498. DOI: 10.1109/ICMLC.2005.1527730

Yan, P. and K. Bowyer, 2007. Biometric recognition using 3D ear shape. IEEE Trans. Patt. Anal. Mach. Intelli., 29: 1297-1308.

DOI: 10.1109/TPAMI.2007.1067 